\title{
Clinical \& Histopathological Features of Ocular Surface Squamous Neoplasia
}

\author{
Dr. Renu Sahay ${ }^{1}$, Dr. Jitendra Kumar ${ }^{2}$, Dr. Arti Kushwaha ${ }^{3}$ \\ ${ }^{\text {I}(D e p a r t m e n t ~ O f ~ P a t h o l o g y, ~ M L B ~ M e d i c a l ~ C o l l e g e ~ / ~ B u n d e l k h a n d ~ U n i v e r s i t y, ~ I n d i a) ~}$ \\ ${ }_{2}^{2}$ (Department Of Ophthalmology, MLB Medical College / Bundelkhand University, India) \\ ${ }^{3}$ (Department Of Ophthalmology, MLB Medical College/ Bundelkhand University, India)
}

\begin{abstract}
:
Purpose: To study various clinical and histopathological features of ocular surface squamous neoplasia. Ocular surface squamous neoplasia (OSSN) represents a rare spectrum of disease ranging from mild dysplasia to carcinoma in situ to invasive squamous cell carcinoma of the ocular surface involving the conjunctiva and the cornea, occurring usually in the interpalpebral area, mostly at the limbus.

Methods And Materials: This study is conducted on 14 patients who underwent surgical excision for histopathologically proven cases of ocular surface squamous neoplasia (OSSN) in deptt of ophthalmology of MLB medical college Jhansi over a period of 6 months. In each case a complete medical and surgical taken, ocular examination including location, extent and clinical history was appearance of the lesion was done. The lesions were surgically excised keeping a safe margin of 2-3 $\mathrm{mm}$ of normal appearing conjunctiva and histopathological studies were performed.

Results: The commonest age group involved in our series was 51-60 years accounting for 83.3\%.The mean age being 54.91 years. Male and female were affected in equal ratio. Mean duration of symptoms was 7.79 months. Mean follow up was 20.8 months. All patients had unilateral tumor with equal involvement of right and left eyes. Interpalpebral involvement was seen in all patients. Temporal quadrant was most commonly affected (66\%). In 7 patients (50\%). Lesions were confined to conjunctiva and in rest 7 patients (50\%) growth extended to cornea. Morphologically (41.6\%) patients were leucoplakic, (33.3\%) patients were papilomatous, 2 (16.6\%) patients were gelatinous and $1(8.3 \%)$ patients were cystic. Impression cytology was done in 8 patients (75\%) and was co-related with histopathology. Systemic predisposing factors like xeroderma pigmentosa was seen in $1(4.16 \%)$ patients and AIDS in 2(8.33\%) patients. Recurrence of lesions was seen in 2 patients (8.33\%), out of which one underwent resurgery and other was treated with topical mytomycin.
\end{abstract}

Keywords: Cornea, Ocular Surface Squamous Neoplasia, impression cytology, squamous cell carcinoma of conjunctiva

\section{Introduction}

Lee and Hirst (1995) first proposed the term "Ocular surface squamous neoplasia (OSSN)" to encompass the spectrum of dysplasia, carcinoma in situ and squamous cell carcinoma of ocular surface i.e., conjunctiva, limbus and cornea ${ }^{[1]}$.It is the third most common ocular tumor after retinoblastoma and melanoma ${ }^{(1,2)}$. OSSN is more common in elderly males in tropical countries due to over exposure to sun light. Diagnosis of OSSN is mainly based on clinical suspicion and confirmed by histopathological evaluation. Prevalence of OSSN varies from 0.03-1.9 per million population depending on the geographic location ${ }^{[3]}$. Risk factors for OSSN include ultraviolet light exposure, fair skin, human papilloma virus (HPV) infection, human immune deficiency virus (HIV) infection, and cigarette smoking, increased p53 expression, chronic inflammatory diseases of the ocular surface such as mucous membrane pemphigoid, chronic blepharoconjunctivitis, and atopic eczema $\left.^{[1,4}\right]$.Recently, immuno histochemically detectable p53 protein, bcl-2 protein, MIB-1 are being used as markers of proliferative potency having a possible prognostic value. MIB-1 is a monoclonal antibody that recognizes Ki-67 antigen which is a marker of cell proliferation.Bcl- 2 is a proto oncogene which is thought to have a role in oncogenesis by inhibiting programmed cell death and preserving cells from p53 induced apoptosis. p53 gene is a common cellular target in human carcinogenesis and thought to have an important role in cellular proliferation. The interplay between the effects of the mutant p53, absence of bcl-2 expression and enhanced expression of MIB-1 are believed to contribute to the progression of OSSN.

\section{Clinical Features}

OSSN typically presents as a growth on the ocular surface and gives rise to symptoms like foreign body sensation, redness or irritation and rarely, diminution of vision due to high astigmatism or involvement of visual axis. It usually starts in interpalpebral conjunctiva and then grows and straddles the limbus and then may or may not involve the cornea. OSSN lesions mostly are slightly elevated and have a pearly grey appearance 
with tufts of vessels commonly known as sentinel vessels, with or without well-defined borders [fig-1]. Regional lymphadenopathy (preauricular nodes, submandibular nodes and deep cervical) should be looked for.

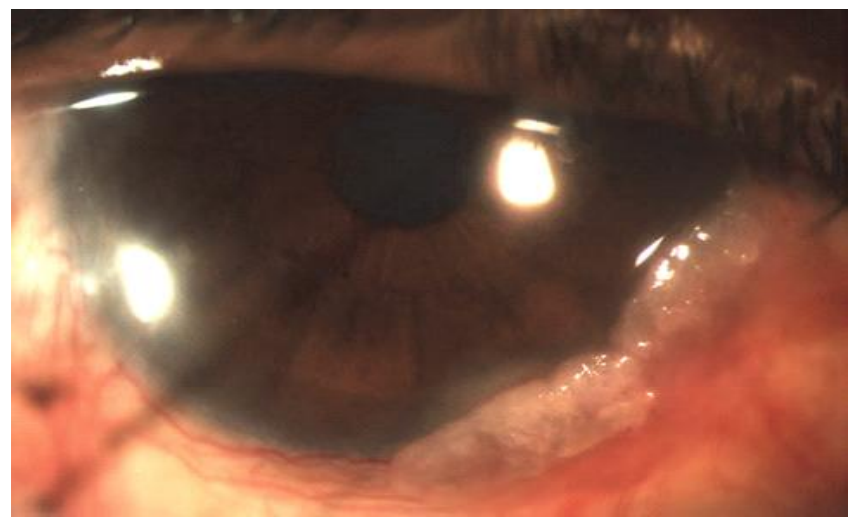

Figure 1 - OSSN seen as a limbal growth

\section{Morphological Classification}

Conjunctival Lesions

1. Gelatinous- Circumscribed gelatinous lesions are the most common. The nodular type is rapidly growing with a high incidence of metastasis to adjacent lymphnodes. The diffuse type is the least common and in the early stages presents as persistent redness of the conjunctiva are slow growing and mimic chronic conjunctivitis. It is difficult to differentiate between benign and malignant lesions in these cases.

2. Leucoplakic- These are usually pre invasive.

3. Papiliform- These type typically are exophytic, strawberry like, with a stippled red appearance corresponding to its fibro vascular core. They are clinically benign.

\section{Corneal Lesions}

Corneal OSSN lesions are pre invasive, with a mottled ground glass sheet appearance which is opalescent. They have sharply defined fimbriated borders, the convex leading edge spreads in an arc away from the limbus and often white dots are present over the grey epithelium. They are usually avascular. These lesions are typically indolent, slow growing and prone to recurrence.

\section{Histopathology $(1,5,6,7)$}

Papilloma - seen as papillary fibrovascular fronds covered by acanthotic epithelium. Adult papillomas show dysplastic features like nuclear enlargement and hyperchromatism, increased nuclear to cytoplasmic ratio, loss of polarity etc.

- Preinvasive lesions are classified as mild, moderate or severe dysplasia, depending on the degree of involvement.

- CIN grade 1 [mild dysplasia] - dysplasia confined to basal [lower] one-third of epithelium

- $\quad$ CIN grade 2 [moderate dysplasia] - extends into middle third of epithelium [Fig 2]

- CIN grade 3 [severe dysplasia] - extending into upper third of epithelium. The basement membrane however is intact. Full thickness dysplasia of the epithelium as in cervical neoplasia is also referred to as 'carcinoma in situ' [Fig 3]. Evidence of chronic inflammation may be seen in substantia propria.

- Invasive OSSN / Squamous cell carcinoma- shows nests of neoplastic cells that have penetrated the epithelial basement membrane and spread into the underlying stroma [Fig4,5].

- Tumor cells may be well-differentiated or ill-differentiated. Two types of cells may be seen interspersed along with squamous cells.

[1] Spindle cells

[2] Mucoepidermoid cells

- The latter is notorious for intraorbital extension as well as early recurrence if left untreated. 
Fig-2

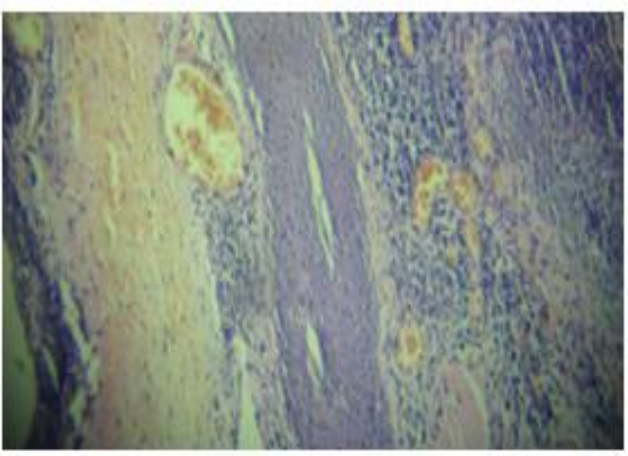

Figure 2- Histopathological section showing moderate dysplasia of adjacert squamous epithelium; H\&E stain.
Fig-3

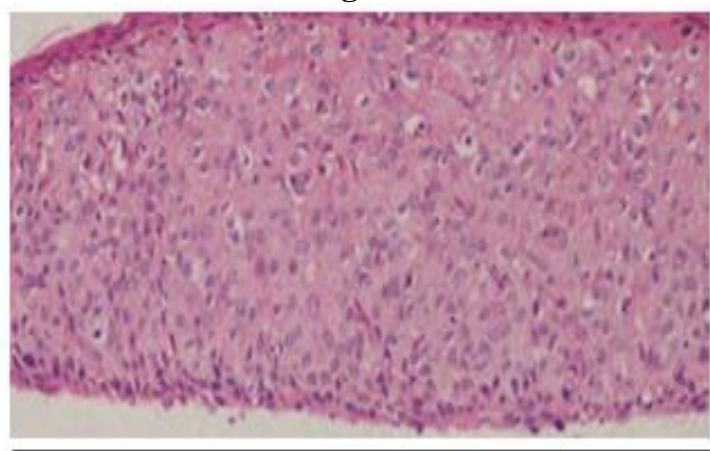

Figure 3 - Carcinoma-in situ - Histopathology section demonstrating dysplastic cells involving the whole thickness of epithelium. The basement membrane is intact.

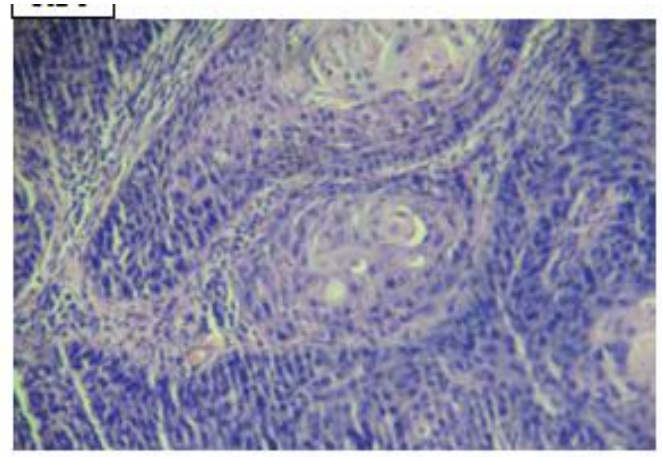

Figure 5- Histopathology section showing malignant squamous cells and foci of epithelial keratin pearls; $\mathrm{H} \& \mathrm{E}$ stain.

Diagnosis - A high index of clinical suspicion is necessary. An incision / excision biopsy is considered the 'gold standard' for diagnosis and planning further treatment. Impression cytology with Biopore membrane has reasonably good accuracy but can miss about $20 \%$ of cases. However it has a definite role in follow-up of lesions, after primary treatment, whether surgical or topical chemotherapy. $5,8,9$

\section{Materials \& Methods}

14 patients over a period of 6 months with histopathologically proven OSSN were reviewed. In each case a complete medical and surgical taken, ocular examination including location, extent and clinical history was appearance of the lesion was done. The lesions were surgically excised keeping a safe margin of 2-3 mm of normal appearing conjunctiva. Cryotherapy was applied to the resected conjunctival margin and limbus with rapid freeze and slow thaw technique. If scleral involvement was seen, partial lamellar sclerotomy was done and the scleral bed was treated with absolute alcohol. Surgical reconstruction if required was done with conjunctival autograft. After initial epithelial healing, patients were recommended to have regular examinations in every 2 months for 1 year and annually thereafter.

\section{Results And Discussion}

Clinically, the diagnosis of OSSN is suspected by the appearance of epithelial changes of the ocular surface. Slit-lamp examination shows gelatinous, leukoplakic, papilliform or nodular lesions. Histopathological evaluation is essential for the definitive diagnosis and also to differentiate the three lesions in the spectrum of OSSN i.e., epithelial dysplasia, carcinoma in situ and invasive squamous cell carcinoma. Though OSSN is considered as a localized, slow growing lesion with low metastatic potential, intraocular, intra orbital and distant metastasis do occur rarely. OSSN has intraocular and orbital extension rate of $4 \%$, regional and distant metastasis rate of $1.2 \%$, and a mortality rate of $0.8 \%$. Sites of metastasis include the preauricular, 
submandibular, and cervical lymph nodes, the parotid gland, lungs, and bones. Regional lymph node involvement precedes the development of distant metastases. ${ }^{[10]}$ The main cause for metastasis is delay in diagnosis and treatment. ${ }^{[3]}$ Surgical excision with "wide margin, no touch" technique is currently the best established form of treatment. Nevertheless, recurrences of these lesions are common after surgical excision, depending on the involvement of the surgical margins. Recurrence rates following excision of OSSN alone range from 15 to $52 \%$, with an average of $30 \%$. Recurrence rate is $5 \%$ when the surgical margins are free and $53 \%$ when the surgical margins are involved. ${ }^{[11]}$ Adjunctive therapy to reduce recurrence includes intra operative cryotherapy and brachytherapy, postoperative topical chemotherapy using mitomycin C, 5-FU and interferon alfa-2.

\section{References}

[1]. Lee GA, Hirst LW. Ocular surface squamous neoplasia. SurvOphthalmol 1995; 39: 429-50

[2]. Lee GA, Hirst LW. Incidence of ocular surface epithelial dysplasia in metropolitan Brisbane: a 10 year survey. Arch Ophthalmol.1992; 110:525-27

[3]. Sun EC, Fears TR, Goedert JJ. Epidemiology of squamous cell conjunctival cancer. Cancer Epidemiol Biomarkers Prev 1997;6:73-7

[4]. Grossniklaus HE, Green WR, Luckenbach M, Chan CC. Conjunctival lesions in adults. A clinical and histopathological review. Cornea 1987; 6: 78-116.

[5]. Pe'er J. Ocular surface squamous neoplasia. Ophthalmol Clin North Am. 2005;18(1):1-13.

[6]. Basti S, Mascai MS. Ocular surface squamous neoplasia. Cornea 2003;22(7):687-704.

[7]. Kiire CA, Srinivasan S, Karp CL. Ocular surface squamous neoplasia. IntOphthalmolClin. 2010;50(3): 35-46

[8]. Tole DM, McKelvie PA, Daniell M. Reliability of impression cytology for the diagnosis of ocular surface squamous neoplasia employing the Biopore membrane. Br J Ophthalmol.2001;85:154-158.

[9]. McKelvie PA, Daniell M. Impression cytology following mitomycin therapy for ocular surface squamous neoplasia. Br $\mathbf{J}$ Ophthalmol.2001;85:1115-19.

[10]. Grossniklaus HE, Green WR, Luckenbach M, Chan CC. Conjunctival lesions in adults. A clinical and histopathological review. Cornea 1987;6:78-116

[11]. Erie JC, Campbell RJ, Liesegang TJ. Conjunctival and corneal intraepithelial and invasive neoplasia. Ophthalmology 1986;93:176-83 